ÉGYPTE monde arabe

\section{Égypte/Monde arabe}

4 | 2007

Figures de la santé en Égypte

\title{
Folkore et éducation populaire
}

Le thème de la santé, des maladies et de l'hygiène dans le Dictionnaire des coutumes, des traditions et des expressions égyptiennes de Ahmad Amîn

\section{Emmanuelle Perrin}

\section{(2) OpenEdition}

\section{Journals}

Édition électronique

URL : https://journals.openedition.org/ema/1763

DOI : 10.4000/ema.1763

ISSN : 2090-7273

Éditeur

CEDEJ - Centre d'études et de documentation économiques juridiques et sociales

Édition imprimée

Date de publication : 31 décembre 2007

Pagination : 103-125

ISBN : 2-6905838-43-4

ISSN : $1110-5097$

Référence électronique

Emmanuelle Perrin, «Folkore et éducation populaire », Égypte/Monde arabe [En ligne], 4 | 2007, mis en ligne le 31 décembre 2008, consulté le 07 juillet 2022. URL : http://journals.openedition.org/ema/1763 ; DOI : https://doi.org/10.4000/ema.1763 
Emmanuelle Perrin

\title{
FOLKLORE ET ÉDUCATION POPULAIRE
}

\author{
LE THÈME DE LA SANTÉ, DES MALADIES ET DE L'HYGIÈNE \\ DANS LE DICTIONNAIRE DES COUTUMES, DES TRADITIONS \\ ET DES EXPRESSIONS ÉGYPTIENNES D'AHMAD AMÎN
}

\begin{abstract}
La vraie question n'est pas de savoir si le contact d'un bec de pic guérit les maux de dents, mais s'il est possible, d'un certain point de vue, de faire « aller ensemble » le bec de pic et la dent de l'homme (congruence dont la formule thérapeutique ne constitue qu'une application hypothétique, parmi d'autres) et, par le moyen de ces groupements de choses et d'êtres, d'introduire un début d'ordre dans l'univers; le classement, quel qu'il soit, possédant une vertu propre par rapport à l'absence de classement.
\end{abstract}

Claude Lévi-Strauss, La Pensée sauvage.

L e Dictionnaire des coutumes, des traditions et des expressions égyptiennes (Qâmûs al-'âdât wa-l-taqâlîd wa-l-ta'âbîr al-misriyya), publié en 1953 par I'homme de lettres Ahmad Amîn (1886-1954), compte parmi les premiers ouvrages consacrés à la description des mœurs et des coutumes égyptiennes ${ }^{1}$. Son auteur appartient à la génération des penseurs et des écrivains qui dominèrent la vie intellectuelle et littéraire de l'entre-deux-guerres. Son œuvre s'inscrit dans le prolongement du mouvement de renaissance culturelle de la Nahda et du courant du réformisme musulman. "Artisan du progrès » engagé au service des idéaux de la réforme qu'il concevait d'abord en termes de culture, Ahmad Amîn se donna une mission et une responsabilité d'éducateur. II voyait notamment la diffusion de l'instruction comme un instrument de progrès social.

En composant ce dictionnaire, Ahmad Amîn procède à la formalisation du «folklore » égyptien en consignant des croyances, des pratiques

1. Les références des articles cités sont celles de l'édition originale. 
et des savoir-faire relevant de traditions orales non codifiées. L'analyse de cet ouvrage permet ainsi d'étudier l'élaboration d'un savoir sur les mœurs et les coutumes et d'aborder la construction de la notion de tradition et du domaine du folklore. Ahmad Amîn donne un motif historique à la collecte des traditions, tout en affirmant le caractère national du mode de vie dit traditionnel ou populaire. Il légitime par conséquent l'étude de la culture dite populaire en l'intégrant à la définition de l'identité égyptienne, comme creuset et conservatoire de celle-ci. II aborde parallèlement nombre d'usages d'un point de vue critique, sous la forme d'une «pédagogie dénonciatrice des erreurs»(Collet, 1987, p. 87). Cette perspective normative, révélatrice des ambitions éducatives du Dictionnaire, contribue également à la définition - ou à l'invention - d'un folklore. Elle témoigne du regard porté par les lettrés, à la fois collectionneurs et pourfendeurs, sur les pratiques dites populaires.

En s'attachant plus précisément aux thèmes de la santé, des maladies et de l'hygiène, il s'agit d'étudier comment et dans quelle perspective ceux-ci se constituent en un objet ethnographique ou folklorique.

Des notations sur la santé, les maladies, I'hygiène et les pratiques médicales des Égyptiens interviennent régulièrement dans le Dictionnaire, dans une proportion qui n'est pas loin de représenter un article sur quatre (125 articles sur 513). Dans la table des matières, outre un article de trente-quatre lignes intitulé «les maladies»( "Al-amrâd»), douze autres articles ont pour titre le nom d'une maladie ou d'une affection. Il s'agit des infirmes ("Ashâb al-'âhât »), de la syphilis («Al-balâ »), de la nausée ( Al-taqrîfa », "Al-gaza' »), de la gale («Al-jarab »), de la fièvre ( Al-hummâ »), des " vers de nez » («Dûdat al-anf »), d'un ver parasite ( Al-saratân »), de la migraine ( $\mathrm{Al}$-sudâ'»), de la teigne ( $\mathrm{Al}$-qurâ'»), des abcès et des bubons ( Kubba ») et du choléra ( $\mathrm{Al}$-kûlîrâ »). On relève également cinq articles dont les titres concernent le traitement des maladies : I'hôpital ( Al-quchlâq ») , la cautérisation par le feu et I'application d'un pois chiche dans une entaille faite sur la peau ( Al-hummusa wa-I-kayy bi-I-nâr »), la médecine " populaire » ou les remèdes de bonne femme ( Al-ruqa »), les «bols d'effroi » ("Tâsit al-khadda »), la cervelle d'âne (« Mukhkh al-himâr »). Un article est intitulé "la propreté » («Al-nazâfa »), et trois autres titres se rapportent à la vermine : les puces («Al-barâghîth»), les punaises ( Al-baqq )), les poux, les puces, les moustiques et les punaises ( Al-qaml wa-I-burghûth wa-I-ba'ûd wa-l-baqq »).

2. Selon Ahmad Amîn, le terme quchlâq employé à la place de mustachfâ pour dénommer l'hôpital dérive d'un mot turc désignant les casernes de l'armée. 


\section{L'INVENTAIRE DES MALADIES DES ÉGYPTIENS : LA RÉALITÉ D'UN ÉTAT SANITAIRE DÉSASTREUX}

Une trentaine de maladies sont inventoriées dans le Dictionnaire. Les maux et les affections les plus souvent cités sont les infirmités, avec notamment la cécité (dix articles), les maladies de peau (dix articles), la fièvre (six articles), les ophtalmies (cinq articles), les maladies vénériennes (quatre articles), les nausées (quatre articles), les maladies parasitaires (trois articles) et la lèpre (trois articles). Se trouvent également mentionnés, avec deux occurrences chacun, le cancer, le choléra, les diarrhées, la dysenterie, les maladies mentales, la migraine, les maladies nerveuses, les rhumatismes, la variole et les "vers de nez ». Enfin, sont signalés une seule fois le diabète, les évanouissements, les maladies du foie, les hémorragies, les hémorroïdes, les maladies imaginaires, les douleurs du nerf sciatique, la paralysie, la peste, les maladies de poitrine, les piqûres de scorpions, la teigne, la toux, la tuberculose, la typhoïde et le typhus.

Les maladies apparaissent comme un point de comparaison entre les Égyptiens et les autres peuples. Ahmad Amîn souligne le fait que certaines affections sont particulièrement répandues en Égypte, et parfois même de manière plus importante qu'ailleurs, comme il le note au sujet des infirmes. Il indique par exemple que les Turcs désignaient les Égyptiens par l'expression méprisante de "paysans aveugles» car les aveugles auraient été plus nombreux en Égypte qu'en Turquie. II rapporte également que des voyageurs européens constatent qu' « en marchant dans les rues du Caire, on croise vingt aveugles, dix borgnes et vingt personnes aux yeux rouges et purulents » (DC, «Al-amrâd», p. 70-71) ${ }^{3}$. La propagation de l'ankylostomiase et de la bilharziose parmi les paysans expliquerait la faiblesse du nombre de jeunes Égyptiens aptes au service militaire en comparaison avec leur proportion dans les pays européens ${ }^{4}$. L'auteur explique également que, du fait de la grande crainte qu'inspirait la conscription, « on voyait rarement de jeunes gens bien portants, la plupart, au contraire, avaient les dents cassées, un doigt coupé, les yeux infectés ou étaient aveugles afin d'échapper à l'enrôlement »(DC, "Al-qur’a / al-tajnîd / al-jihâdiyya », p. 323). Parmi les maladies courantes, il

3. L'article ne précise pas l'époque de cette observation. Le problème de la datation et de l'actualité des usages et des faits décrits se pose régulièrement à la lecture du Dictionnaire. De telles imprécisions et ambiguïtés sont sans doute entretenues par le fait que le domaine des traditions paraît immémorial et atemporel. Cependant cet article semble bien traiter des maladies des Égyptiens à l'époque de la rédaction du Dictionnaire, soit la fin des années 1940 et le début des années 1950.

4 Dans les années 1920, seuls 4,5\% des 90000 jeunes gens en âge d'effectuer leur service militaire étaient déclarés aptes. Les autres étaient refusés en raison de leur faiblesse due aux maladies parasitaires. Voir Chiffoleau, 1997, p. 101. 
énumère les ophtalmies, les maladies parasitaires, les diarrhées, la dysenterie, la gale, la typhoïde et le typhus, ces deux derniers étant décrits comme des maladies endémiques. L'éruption des «boutons du Nil » (habb al-Nîl, hamû al-Nîl) au moment de la crue du Nil constitue en outre une affection particulière à I'Égypte. Les remarques de Ahmad Amîn reflètent l'état sanitaire du pays à cette période. L'ankylostomiase ${ }^{5}$ touchait $30 \%$ et le trachome ${ }^{6} 90 \%$

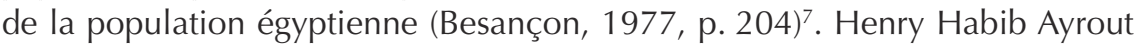
indique qu'à la fin des années 1930, les borgnes et les aveugles représentaient $1 / 23^{e}$ de la population (Ayrout, 1938, p. 100). Quant à la bilharziose, $75 \%$ des ruraux en étaient atteints (Besançon, 1977, p. 100) ${ }^{8}$. L'espérance de vie était de 40,7 ans en 1955 (Boustani et Fargues, 1990, p. 126) ${ }^{9}$.

Dans sa présentation des maladies, Ahmad Amîn relève également des expressions, des insultes, des proverbes et des chansons liés à certaines d'entre elles. II signale par exemple les injures « que la peste l'étouffe ! " (gâ't-hu kubba), "que la diarrhée l'emporte!» (gâ't-hu al-batn). La belle-mère est comparée à la fièvre, et la sœur du mari à une piqûre de scorpion. Diverses notations se rapportent par ailleurs au «statut» social des malades et des infirmes et aux croyances qui les entourent. La visite des malades apparaît ainsi comme l'une des manifestations de la solidarité et des liens qui unissaient les habitants d'un même quartier. La guérison d'une maladie donne lieu à une fête. Ahmad Amîn indique que l'exercice de certaines professions semble réservé aux infirmes. Ainsi, la mémorisation et la récitation du Coran déterminaient presque toujours la situation et I'avenir des aveugles. II constate que la plupart des marchands de journaux sont boiteux et que, dans I'oasis d'al-Kharga, les porteurs d'eau sont généralement aveugles. II relève la croyance selon laquelle rencontrer un borgne ou un teigneux le matin porte malheur. Au sujet des présages liés à l'orientation des maisons, l'auteur note qu'une habitation dont la porte s'ouvre à l'est apparaît comme un gage de santé et de vitalité.

5. Maladie provoquée par un ver parasite (nématode) et caractérisée par une anémie, due à des pertes continues de sang dans les intestins, des troubles digestifs et parfois des œdèmes.

6. Le trachome est une conjonctivite contagieuse et chronique, d'origine bactérienne, endémique dans certains pays chauds et pouvant entraîner la cécité.

7. Selon Chiffoleau (1997, p. 100), I'ankylostomiase touchait environ $50 \%$ de la population.

8. La bilharziose est une maladie parasitaire due à la pénétration dans la peau et le sang de vers appartenant à la famille des trématodes (bilharzies) et qui entraîne une hématurie chronique (sang dans l'urine)

9. En 1950, la mortalité infantile était de 200 pour 1 000. Dans les années 19851990, I'espérance de vie atteignait de 59,9 ans (58,4 pour les hommes et 61,4 ans pour les femmes). 


\section{LES CAUSES DES MALADIES}

\section{Le fonctionnement du corps humain et les causes des maladies}

Ahmad Amîn met en cause certaines représentations du corps et de l'étiologie des maladies qu'il juge par trop surnaturelles. Le fonctionnement du corps lui paraît méconnu. Ainsi, dans l'article «Ventre » («Batn »), il note qu' « avant que le peuple $\left(a l-c h a^{\prime} b\right)$ ne s'instruise (yatathaqqafu), le malade ne distinguait pas ses organes et disait "j'ai mal au ventre" qu'il ait mal à l'estomac, aux intestins, au foie ou aux deux. Quand l'éducation hygiénique (al-thaqâfa al-sihhiyya) progressa, le mot [ventre] disparut et les gens commencèrent à dire "j'ai mal à l'estomac ou au foie ou autre chose" » (DC, "Batn», p. 90). Le corps humain apparaît également largement soumis à des influences surnaturelles. On constate ainsi que, à l'exception de l'article "Ventre », tous les autres articles ayant un titre en rapport avec une partie du corps ou un phénomène physique traitent de croyances magiques, de l'action des démons ou de la divination. Des parties du corps comme les cheveux, les ongles ou le prépuce servent à l'ensorcellement. Les bâillements, causés par les démons, sont de mauvais augure. L'auteur cherche à corriger de telles conceptions en expliquant par exemple que «les fourmillements dans les jambes, les clignements des paupières, les picotements des mains sont des phénomènes naturels dans lesquels la mentalité superstitieuse (al-'aql al-khurâfî) voit le signe de quelque chose » (DC, "Tanmîl al-rijl », p. 128). Le corps subit également l'emprise d'esprits, la maladie étant alors perçue comme l'un des symptômes de la possession.

Ahmad Amîn dénonce également les origines surnaturelles attribuées aux maladies. Il déplore que le mauvais œil en soit considéré comme une cause, y compris de la fièvre. Les djinns sont responsables de maladies que déclenchent leur colère ou la possession : " [les Égyptiens] croient que la cause des maladies est une jinniyya noire qui possède l'homme ou la femme. Celui qui est possédé ne pourra se la concilier que par le zâr » (DC, « Jinn », p. 142). Ils passent également pour échanger les enfants sains contre des enfants malades ${ }^{10}$. Les maladies peuvent aussi apparaître comme des châtiments : I'argent mal acquis, par le vol ou la corruption, nuit à son possesseur et à son entourage qui se trouvent atteints de maladies graves ou d'infirmités. Au sujet des maladies mentales, jugées par ailleurs peu répandues en Égypte ou liées à la consommation de drogues, l'auteur constate la confusion entre sainteté et folie. Quant aux maladies vénériennes, il relève la croyance selon laquelle celles-ci seraient dues à une peur soudaine ou au froid : « [Les Égyptiens] ne croient pas que les rapports

10. Ahmad Amîn dénonce à ce sujet les rituels accomplis autour de la serrure de bois de la mosquée des Awlâd al-'Annân, qui garantirait la restitution des enfants sains, ainsi que l'imposture entretenue par le personnel de la mosquée (DC, «Al-dabba », p. 267). 
honteux en soient la cause mais une peur ou un froid intense. C'est pourquoi ils n'ont bien souvent pas de honte à les évoquer ni à les attraper » (DC, «Alamrâd», p. 71).

Ahmad Amîn oppose des explications scientifiques et médicales aux croyances qu'il relève. Les articles portant sur les thèmes de la santé et de l'hygiène prennent de ce fait un certain caractère éducatif. Ainsi en est-il de la fièvre : « les expérimentations des savants ont montré qu'elle [était causée] par différents microbes », un examen médical permet d'en diagnostiquer chaque type, « cependant le commun du peuple ('âmma) croit qu'elle est une espèce de djinn qui possède les humains et les rend malades » (DC, «Al-hummâ », p. 181). Les Égyptiens prétendent qu'une peur soudaine cause des maladies nerveuses graves et la syphilis (al-zuharî) «bien qu'il soit médicalement avéré que [celle-ci] ne se contracte que par des rapports sexuels avec une personne malade » (DC, "Tâsit al-khadda », p. 275). L'auteur explique également que les ophtalmies, très répandues en Égypte, sont provoquées par la poussière, la lumière et la chaleur, ou encore que la teigne provient d'un microbe contagieux qui attaque les cheveux. Au sujet des infirmes, Ahmad Amîn estime qu'ils sont plus nombreux en Égypte qu'ailleurs en raison de la saleté, du recours à la « médecine populaire » (tibb al-ruqa), du manque de confiance dans les médecins et de la négligence des traitements. II explique ainsi que I'hôpital représente un lieu honni et effrayant, où les gens répugnent à envoyer leurs malades, craignant notamment qu'ils y soient maltraités par les médecins et les infirmiers ${ }^{11}$.

\section{Le manque de propreté et d'hygiène}

L'exposé des causes des maladies renvoie dans une large mesure aux problèmes de propreté et d'hygiène, thèmes abordés par une trentaine d'articles du Dictionnaire. Ahmad Amîn propose notamment un classement des nations orientales selon leur propreté, au premier rang desquels viennent les Turcs, suivis des Libanais, des Syriens, des Égyptiens, des Iraniens et des Indiens. Certaines communautés se distinguent des Égyptiens par leur propreté ou celle de leurs maisons, comme les Circassiens et les Nubiens. Cinq articles évoquent la propreté des Turcs, qui est bien I'une des rares qualités que leur reconnaisse l'auteur.

La saleté et la poussière sont présentées à plusieurs reprises comme des vecteurs de maladies et d'infirmités. Différents articles évoquent la saleté des logements, des rues et des quartiers : manque de propreté des maisons, y compris celles des notables, notamment dans les campagnes, "situation très misérable» (DC, «Al-darâ'ib », p. 268) des pauvres et des paysans, saleté des

11. Dans son autobiographie, Ahmad Amîn évoque la mort de son frère cadet, à l'âge de seize ans. Alors qu'il était atteint de fièvre typhoïde, ses parents refusèrent de l'envoyer à l'hôpital, malgré l'insistance du médecin et de l'auteur (Amîn, s.d., p. 88-89). 
quartiers populaires (baladî) du Caire, épluchures et eaux usées jetées dans les rues $^{12}$. Une notation concerne le manque d'hygiène corporelle : les enfants et les pauvres passent plusieurs jours sans se nettoyer le visage et ne lavent pas leurs vêtements. Plusieurs articles décrivent les conditions sanitaires misérables dans lesquelles vivent les paysans : saleté de leur environnement et de leur logement, prolifération des poux et des puces, pauvreté de leur nourriture, utilisation d'eau non potable. La propagation des maladies parasitaires serait notamment due à la saleté de l'eau que les paysans consomment. Leur manque de propreté se manifeste selon l'auteur par le fait qu'ils portent rarement de chaussures ${ }^{13}$, $q u^{\prime}$ « ils mangent des radis et des poireaux après les avoir lavés dans l'eau sale des canaux et qu'il boivent l'eau du Nil sans la filtrer. Peut-être iront-ils rapidement de l'avant sur la voie de la propreté » (DC, «Al-nazâfa », p. 398).

\section{Les insectes et la vermine}

En relation avec les problèmes de propreté et d'hygiène, Ahmad Amîn consacre trois articles aux insectes qui parasitent I'homme ou lui transmettent des maladies, les poux, les puces, les moustiques et les punaises. Il convient de souligner le caractère pédagogique de ces articles. Ainsi, alors qu'il relève que les paysans croient que les poux proviennent de la sueur, I'auteur explique que leur prolifération est due au manque de propreté (DC, «Al-qaml wa-l-burghûth wa-I-ba'ûd wa-I-baqq ", p. 328). Il constate la diminution des puces, qui constituaient l'un des fléaux de l'Égypte, avec le développement de l'hygiène et I'utilisation d'antiseptiques. II indique que la vermine est un vecteur de maladies comme la gale et relève à trois reprises que la nourriture est exposée aux mouches. Il observe par ailleurs que les Égyptiens fabriquaient des amulettes pour se préserver des punaises et donne la formule d'un charme (ta'wîdha) destiné à chasser les puces, les punaises et les fourmis d'une pièce (DC, "Al-baqq ", p. 92). L'auteur cite par ailleurs un poème de l'écrivain libanais Ahmad Fâris Shidyaq (1804-1887) sur les puces (DC, "Al-barâghîth », p. 8384 ) et consigne quelques expressions et proverbes, par exemple « comme les puces du pont, elle sont microscopiques et impudentes ${ }^{14}$ " (zayy barâghîth alqantara, qilla wi zantara) (Ibid.), " comme la punaise, elle enfante cent [petits] et dit : quelle maigre progéniture ${ }^{15}$ ! » (zayy al-baqq tiwled meyya we teqûl yâ qillat al-zurreyya) (DC, «Al-baqq», p. 92).

12. Ahmad Amîn signale à ce sujet l'expression « dastûr! " par laquelle on prévient les passants et les djinns que l'on va jeter de l'eau sale dans la rue.

13. L'auteur évoque par ailleurs la création d'un comité pour interdire de marcher pieds nus. Voir DC, «Al-hufâ », p. 172.

14. Selon la traduction proposée par Tadié, 2002, p. 196.

15. D'après la traduction proposée par Tadié, 2002, p. 390. 


\section{La contagion des maladies}

À plusieurs reprises, Ahmad Amîn met en évidence le mécanisme de la contagion des maladies, notamment la contamination par l'eau. Il explique ainsi que l'eau des puits des maisons, principalement utilisée pour la toilette et la vaisselle, est contaminée par les infiltrations des latrines, ce qui cause aux habitants des maux petits et grands. L'article portant sur les bassins d'ablution des mosquées ("Al-mîda ») dresse un véritable réquisitoire contre ces installations dont l'eau est contaminée par les infiltrations des latrines ou par les microbes d'un homme malade. S'y ajoutaient les risques de noyade. L'auteur s'en réfère à sa propre expérience puisque, enfant, il faillit se noyer dans I'un d'eux ${ }^{16}$. Il évoque les appels des réformateurs en faveur de leur remplacement par des robinets et explique que l'on reprocha à Muhammad 'Abduh d'avoir fait disparaître la baraka d'al-Azhar quand il décida d'un tel changement. C'est une véritable guerre que durent livrer les robinets comme en témoigne l'emploi de termes militaires : "L'offensive des robinets contre les bassins d'ablution dura jusqu'à la défaite [de ces derniers], car les robinets étaient plus sains et plus propres » (DC, "Al-mîda», p. 392). Ahmad Amîn rapporte également que les eaux de l'ancien canal qui traversait Le Caire étaient polluées par les ordures, les cadavres d'animaux et les prépuces des enfants circoncis qu'y jetaient les gens, si bien que tout ceux qui en buvaient tombaient malades. De plus, les insectes, en particulier les moustiques y proliféraient au moment de la décrue. L'auteur approuve pour toutes ces raisons son remblaiement par le gouvernement.

Des considérations d'hygiène apparaissent également au sujet des écoles coraniques. Ahmad Amîn note que celles-ci se trouvaient bien souvent dans des endroits insalubres, manquant de lumière et de soleil, et à proximité des latrines de la mosquée. À quoi s'ajoutait la contagion des maladies car les enfants mangeaient à la main dans les mêmes plats et ceux qui étaient malades contaminaient les autres. De même, il explique les mesures prophylactiques visant à interdire la coutume des embrassades et des étreintes : "La civilisation a combattu les embrassades en période d'épidémie car elles sont une cause de contagion, elle a interdit que les jeunes baisent par respect les mains des personnes plus âgées et a limité les embrassades aux baisers d'amour » (DC, "Al-qubla», p. 319). II souligne également, outre les méfaits du tabac, la nocivité des cigarettes fabriquées à partir de mégots, contaminés par les microbes qui se trouvaient par terre et par ceux dont le premier fumeur était porteur.

16. Ahmad Amîn relate également cet accident dans son autobiographie, comme I'un de ses trois premiers souvenirs (Amîn, s.d., p. 25). 


\section{Le climat et les saisons}

Six articles évoquent l'incidence du climat et des saisons sur la santé et les maladies. L'auteur explique ainsi que les maladies du foie sont liées à la chaleur qui fait boire beaucoup d'eau et que les ophtalmies sont provoquées par la lumière et la chaleur. Des dictons illustrent par ailleurs les conséquences des rigueurs de I'hiver ou mettent en garde contre la consommation de mulûkhiyya (corette potagère) : "Qui mange de la mulûkhiyya pendant le mois d'abîb [début juillet à début août] doit faire appel au médecin pour [ses maux] d'estomac ${ }^{17}$ " ("Min yâkol mulûkhiyya fî Abîb yigîb li-batn-hu tabîb ») (DC, «Al-chuhûr al-qubtiyya», p. 253-254). Des vents chauds et violents, gênant la respiration, soufflent durant les cinquante jours (khamâsîn) qui suivent la fête de chamm al-nasîm. Les Égyptiens sortent ce jour-là pour en respirer les premières brises, croyant ainsi se fortifier contre les maux (churûr) causés par ces vents ${ }^{18}$. Les Égyptiens mettent la " nuit de la goutte » (Îlit alnuqta, soit la goutte qui tombe dans le Nil pour le faire monter), qui a lieu en général le onzième jour du mois de ba'ûna (mi-juin mi-juillet), en relation avec la purification de l'air et la prévention des maladies et des épidémies. Enfin, le moment de l'ouverture du canal du Caire, fin août début septembre, au maximum de la crue du Nil, était considéré comme la période la plus appropriée, selon Ahmad Amîn pour des raisons climatiques, à la pratique de la circoncision.

\section{LE TRAITEMENT DES MALADIES}

Le traitement des maladies fait l'objet d'une description assez détaillée dans le Dictionnaire. En effet, l'auteur évoque les remèdes apportés à vingt des trente-trois affections ou types de maladies recensés ; la majorité d'entre eux, soit dix-huit sur vingt, concerne des prescriptions qui relèvent $d^{\prime}$ une « médecine populaire ».

La " médecine populaire », les remèdes et prescriptions des vieilles femmes (tibb al-ruqa)

Quatre articles évoquent la "médecine populaire », fondée sur les remèdes et prescriptions des vieilles femmes ou tibb al-ruqa, littéralement « la médecine de la quenouille», expression généralement traduite par « remède de

17. Selon la traduction proposée par Tadié, 2002, p. 578.

18. D'où le nom de Chamm al-nasîm, littéralement « respire la brise ». Divers usages prophylactiques, notamment à l'aide d'oignons, consistent aussi ce jour-là à se protéger des esprits que transportent les vents. 
charlatan » ou « de bonne femme ». Un court article du Dictionnaire y est principalement consacré :

Certains prétendent que " al-ruqa » désigne dans le langage des vieilles femmes un morceau de bois sur lequel elles filaient le lin. Cette tâche était assignée aux femmes. Elles se réunissaient autour de la ruqa pour accomplir ce que leur mari ou leur maître leur avaient assigné. Chaque femme décrivait une recette (wasfa) qui avait guéri. Par conséquent, on a appelé la médecine qui se fonde sur les prescriptions des vieilles femmes «tibb al-ruqa ». Des livres ont été écrits à ce sujet. (DC, «Al-ruqa », p. 214)

Dans un article sur les vieilles femmes (al-'ajâ'iz), Ahmad Amîn indique également qu'elles sont « réputées pour les prescriptions populaires (al-wasafât al-baladiyya) qu'elles tiennent de leur expérience et de leurs devancières » (DC, «Al-'ajâ'iz », p. 284). Selon l'auteur, le recours à ce type de remède est en partie responsable du grand nombre d'infirmes que l'on trouve en Égypte. Il illustre également le caractère " hasardeux » de l'éducation que les femmes donnent à leurs enfants : "l'éducation que la mère donne à ses fils n'est pas fondée sur des principes éducatifs, elle est plutôt hasardeuse : si l'enfant est malade, elle a recours à la médecine des vieilles femmes (tibb al-ruqa) » (DC, «Tarbiyat al-atfâl », p. 114-115).

\section{La pharmacopée}

Douze articles signalent I'utilisation médicinale de plantes, parmi lesquelles l'armoise (chîh), une plante appelée barnûf (Conyza dioscoridis), les " graines de baraka » ou graines de la fleur de fenouil, le henné, I'ipéca ('irq al-dhahab), le ladanum (libân dhakar), le lupin (turmus), les graines de réglisse indienne (chechm), la ra'ra' Ayyûb (Pulicaria arabica), la rue (sadab) et la salsepareille ('achba, 'ichba). Deux articles évoquent en outre les marchands de plantes médicinales ('aqâqîr) du marché d'al-Ghûriyya et du quartier d'al-Tarbî'a, au Caire. Le Dictionnaire mentionne également I'utilisation de substances animales comme la cervelle d'âne et les "pierres de scorpion » (hagar al-'aqrab), c'est-à-dire leur lobe (fass) ou glande à venin. Les propriétés curatives ou l'utilisation médicinale de divers aliments sont également signalées : canne à sucre, citron, graines de courge, eau du Nil, huile, mech (un fromage fermenté), racine de mughât (Glossostemon brugieri), oignons, raisin, sel, sucre, viande de canard et de pigeon, vinaigre. Ahmad Amîn relève par ailleurs que les appels des vendeurs de bananes et de fenugrec comportent l'idée de guérison : «La guérison par Dieu, ô banane » (al-chafâ min Allâh yâ mûz), "La guérison par Dieu, ô fenugrec » (al-chafâ min Allâh yâ hilba). Il mentionne en outre I'utilisation médicale de l'amidon, de l'arsenic, de l'encens, du khôl, du plomb, de l'or, du soufre et de diverses pierres. Au total, quelque trente-six drogues se trouvent ainsi répertoriées dans le Dictionnaire. À titre de comparaison et pour 
donner un ordre de grandeur, les membres de la Société de géographie d'Égypte qui, dans les années 1930-1940, souhaitaient compléter les collections de son Musée d'ethnographie par la reconstitution d'un « droguier égyptien » rassemblèrent une centaine d'échantillons (Bovier-Lapierre, 1948, p. 183).

\section{Le recours à la religion et au pouvoir guérisseur des saints}

Ces différents remèdes ne constituent pas les seuls moyens de soigner les maladies. Sept articles témoignent du recours à la religion et au pouvoir guérisseur des saints. La guérison des maladies figure parmi les principales propriétés attribuées aux sourates du Coran. Ahmad Amîn cite également les formules de deux invocations de guérison et fait mention de prières récitées pour la santé des enfants. L'article "Qiyâs al-athar» décrit un procédé particulier mis en œuvre par des fuqahâ'. Dans les campagnes, les malades font porter au faqîh leurs effets personnels avec un peu d'argent. Le faqîh prononce une incantation sur les vêtements pour prédire la guérison et rédige une amulette en fonction. Le recours au pouvoir guérisseur des saints est évoqué dans le Dictionnaire au travers de la pratique des ex-voto ou offrandes votives promises aux saints contre la réalisation de quelque chose, dont la guérison d'une maladie. L'auteur signale également que des sirops, boisson de fête, sont offerts lors de la réalisation d'un vœu de guérison sans toutefois préciser comment et à qui ce vœu est adressé. Un article porte enfin sur la source située à proximité du tombeau de l'imam al-Chafi î. Ahmad Amîn s'attache à démystifier les vertus curatives que les Égyptiens attribuent à la baraka de l'imam.

['Ayyin al-sîra] est une source salée qui coule près [du tombeau] de l'imam Chafi'î. Les Égyptiens croient que celui qui s'y lave guérit de ses maladies, par la baraka de l'imam. En vérité, la source contient des substances chimiques. Grâce à ces substances qui s'écoulent dans l'eau, [celle-ci] guérit certaines maladies, notamment les maladies de peau, de même que la boue dans laquelle se concentrent ces substances. [Les Égyptiens] ont l'habitude de prendre de cette boue pour l'appliquer sur le membre blessé. Elle absorbe la plupart des écoulements nocifs et le malade guérit. (DC, «'Ayyin al-sîra », p. 294)

Des références religieuses apparaissent également dans les formules de politesse échangées avec celui qui prend un traitement, auquel on doit dire "Avec la santé » (bi-l-châfa), ce à quoi il répond "Que Dieu vous donne force et santé » (chafâ-kum Allâh w-'âfâ-kum), ou adressée à celui qui se relève d'une maladie, à qui l'on souhaite d'être récompensé et doté d'une bonne santé ("agr w-'afya »), et qui répond en retour "Que Dieu vous donne de la force » ('âfâ-kum allâh). L'auteur explique la formule «le hammam est gage de santé » (hammâm al-'afya) comme une expression demandant à Dieu de faire en sorte que celui qui se rend malade au hammam en revienne guéri. 


\section{Les pratiques magiques et divinatoires}

Douze articles traitent des pratiques magiques et divinatoires liées au diagnostic et à la guérison des maladies. Trois d'entre eux signalent l'utilisation d'amulettes. Concernant la divination, l'auteur note que des tables divinatoires peuvent être consultées au sujet d'un malade. Il dit avoir assisté à une séance d'invocation des esprits (arwâh), au cours de laquelle un homme sous hypnose révéla aux participants leurs maladies et les moyens d'en guérir. Ahmad Amîn précise que le traitement qui lui a été ainsi prescrit, et qu'il a essayé, n'a pas réussi. Pour soigner les maladies nerveuses et la syphilis (al-zuharî), les Égyptiens utilisent un «bol d'effroi » (tâsit al-khadda), un récipient gravé de dessins et d'inscriptions. Le malade doit boire l'eau exposée durant la nuit dans ce bol. Ahmad Amîn signale que les magiciens récitent une incantation ('azîma) pour soigner les migraines. Deux articles traitent des vers de nez (dûdat al-anf) qui se logeraient dans le nez des enfants, et que l'on fait sortir à l'aide d'incantations. Deux rituels magiques sont précisément décrits. L'un concerne la guérison d'une maladie causée par la colère d'un djinn (DC, « Jinn », p. 428) et l'autre consiste à «traire les étoiles » pour obtenir une eau guérissant les maladies de peau :

Ceux qui feignent la magie placent un sequin vénitien dans de l'eau et s'installent sur un toit la nuit, avec le récipient contenant le sequin et l'eau. Au lever d'une certaine étoile, ils prétendent réciter des incantations dans sa direction et qu'il en descend de l'eau dans le récipient, une eau qu'ils conservent précieusement, et qui, selon leurs allégations, guérit en une seule application toutes les maladies de peau, comme la gale, les maladies vénériennes, les abcès, etc. (DC, "Bunduqî », p. 97)

\section{Les praticiens de la médecine}

Dix articles font mention des médecins (tabîb, pl. atibbâ'). Ahmad Amîn note par exemple que leur activité était autrefois contrôlée par l'inspecteur des marchés (muhtasib) qui pouvait interdire l'exercice de la médecine à un praticien dont les remèdes ne guérissaient pas. II évoque également la concurrence qui existait entre les médecins égyptiens et étrangers. Les Égyptiens croyaient d'avantage à la science des Européens qu'à celle de leurs compatriotes et considéraient les médecins étrangers meilleurs que les praticiens égyptiens, même quand leurs diplômes étaient plus modestes. Si l'auteur souligne le manque de confiance à l'égard des médecins, il signale cependant que les familles aisées leur font appel pour pratiquer la circoncision. L'article consacré au budget annuel d'une famille de condition moyenne vers 1950 mentionne des dépenses liées à la consultation d'un médecin et à l'achat de médicaments.

Aux côtés des médecins, apparaissent les barbiers (mezayyen), les sagesfemmes (dâya), les bédouins dont la spécialité est la cautérisation par le feu, 
les poseurs de ventouses (mekabbâtî), les fuqahâ', les guérisseuses par l'encens, les vieille femmes, les apothicaires ('attâr) et les marchands de plantes médicinales ('aqâqîr). L'exposé des pratiques magiques liées à la guérison des maladies désigne des magiciens, devins et faiseurs d'amulettes.

Une large part des articles traitant de la santé et de l'hygiène, soit cinquante-sept au total, concernent les Égyptiens dans leur ensemble ou les gens. Il ne s'agit donc pas d'une médecine populaire qui serait le fait du commun du peuple ('âmma). La référence à celui-ci n'intervient principalement qu'au sujet de la fièvre et des vers de nez. Ahmad Amîn décrit plutôt une médecine " traditionnelle » ou ancienne qui paraît « populaire » dans la mesure où elle n'est pas savante, c'est-à-dire pratiquée par des médecins.

\section{L'efficacité des traitements}

Au travers des articles qu'il consacre aux thèmes de la santé et de la maladie, Ahmad Amîn consigne et décrit les savoirs et savoir-faire de la médecine " populaire » ou «traditionnelle ». Ce faisant, il apporte certaines précisions sur l'efficacité ou l'absence d'efficacité de ces remèdes. L'auteur adopte une position réservée à l'égard de nombre de ces traitements en les exposant sans en reconnaître ni en démentir l'utilité. II souligne également le caractère douloureux de quelques-uns d'entre eux. II reconnaît cependant les vertus curatives de certains de ces remèdes. D'autres sont enfin présentés comme des croyances ou encore jugés invraisemblables, inefficaces voire meurtriers.

\section{Un jugement réservé, les traitements dont l'efficacité n'est ni validée ni infirmée}

Un certain nombre d'articles mentionnent des prescriptions et des traitements dont l'efficacité médicinale n'est ni reconnue ni démentie par I'auteur. Du canard noir, notamment son foie, est prescrit aux paralytiques, la viande de pigeon est donnée aux convalescents. Le jus de canne à sucre est, tout comme celui du raisin, employé comme fortifiant. Le citron est utilisé contre les nausées, ainsi que l'illustre le proverbe « Un citron dans un pays dégoûtant » (Lamûna fî balad qarfâna) ${ }^{19}$. On remédie également à l'écœurement en accrochant quelque chose de jaune sur la tête du malade de manière à ce que cela lui retombe devant les yeux. Les graines de la réglisse indienne soignent les inflammations oculaires. La gale est traitée avec du sel ou une préparation composée de soufre broyé et de sucre, que I'on absorbe ou que I'on applique en pommade. Du henné dilué avec du

19. Ahmad Amîn explique ce proverbe par le fait que dans un endroit dégoûtant les gens se disputent un citron. Selon le dictionnaire de Hinds et Badawi, il signifie « juste ce dont on a besoin ». 
vinaigre est déposé sur le front d'un malade pour faire baisser la fièvre. Le ladanum est employé contre la toux et le lupin, réduit en poudre et frotté sur le corps, traite les «boutons du Nil ». Le khôl est utilisé pour soigner les yeux, on y ajoute alors du minerai de plomb, du manzût, de I'ipéca, du sucre cristallisé et de l'or pilé d'un sequin vénitien. Ahmad Amîn donne la composition d'une préparation comprenant du jus d'oignon et de I'armoise, servant à traiter les ophtalmies, et qui est aussi donnée à respirer en cas d'évanouissement. L'oignon connaît un usage prophylactique : les Égyptiens en mangent et en respirent en temps d'épidémie, de même qu'ils croient devoir en consommer en arrivant dans un nouveau pays ${ }^{20}$. Le Dictionnaire signale l'emploi de nombreuses pierres aux vertus médicinales, dont une "pierre de sang " (hagar al-damm), sorte de cornaline verte veinée de rouge, portée pour empêcher les hémorragies. De la confiture de graines de courge est donnée en cas d'élévation de la pression sanguine. Un article détaille les différents traitements des maladies vénériennes, qui consistent à absorber de l'huile chaude, à appliquer sur les plaies pour les cautériser un chiffon empli de sel et bouilli dans de l'huile ou à fumer un narguilé de tabac à l'arsenic.

\section{Des traitements douloureux}

Ahmad Amîn mentionne par ailleurs le caractère douloureux ou pénible de certains remèdes. Un tel aspect justifie même la présence d'un article sur la teigne dans le Dictionnaire. "II n'y aurait pas lieu d'évoquer cette maladie courante qu'est [la teigne] si ce n'est qu'ils la soignent parfois avec des traitements brutaux» (DC, "Al-qurâ'», p. 322) : on enduit de goudron mêlé à quelques remèdes la tête du malade puis on la recouvre d'une toque. Au bout d'une semaine, on arrache la toque avec le goudron. L'auteur signale en conclusion de son article qu'avec les progrès de la médecine, il est désormais possible de soulager les souffrances des teigneux et de les soigner avec des pommades à la pénicilline ou au sulfate. Il décrit aussi le «traitement abominable » que s'infligeaient les Soudanais atteints par la gale en frottant leurs plaies jusqu'au sang avec un tesson pour y mettre du sel. La cautérisation par le feu, employée pour le soin des rhumatismes et des douleurs du nerf sciatique, est également citée comme un remède particulièrement pénible, ce qu'illustre le proverbe arabe "la cautérisation est le dernier remède $»^{21}$. L'auteur indique que ce traitement dont on peut se passer grâce aux médicaments modernes, n'est plus pratiqué bien que certains disent qu'il réussit là où la médecine moderne a échoué.

20. Ce dernier point évoque un proverbe relatif à la capacité d'adaptation : "Quand tu arrives dans un pays, nourris-toi de ses oignons », Tadié, 2002, p. 234.

21. "Âkhir al-dawâ' al-kayy» que l'on peut également traduire par "Aux grands maux, les grands remèdes ». 


\section{Des remèdes efficaces}

Les vertus curatives de certains traitements sont parfois reconnues par I'auteur. Il confirme ainsi que le fait de sucer de la canne à sucre (qasab) fortifie les gencives et blanchit les dents. Au sujet du citron, il indique que l'étymologie du mot d'origine persane «banzahîr » (en français bézoard) signifie contrepoison et souligne qu'il s'agit d'un fruit bénéfique, « riche en vitamine A comme l'ont prouvé les analyses modernes ${ }^{22} »(\mathrm{DC}$, «Al-laymûn al-saghîr », p. 350). L'auteur reconnaît également les vertus apaisantes de l'encens : "il est vrai que l'encens apaise les nerfs et que celui qui est encensé ressent effectivement ses nerfs se calmer. C'est la raison pour laquelle l'encens est associé à la récitation du Coran dans les mosquées et à celle des prières dans les églises »(DC, "Al-bakhûr », p. 80). Ceci explique son efficacité médicinale : "les femmes et certains hommes se rendent auprès de femmes célèbres en Égypte pour y être encensés. Comme les maladies des femmes sont souvent imaginaires ou nerveuses, I'encens leur est bénéfique » (Ibid., p. 81). L'efficacité de la « pierre de scorpion » (hagar al-'aqrab) pour soigner leurs piqûres, bien que décrite comme une croyance, est confirmée par des médecins. "Ils sont nombreux à attester, et parmi eux des médecins, que [ces] lobes sont efficaces en cas de morsure de scorpion» ("Istabl 'Antar», p. 48-49) ; I'auteur donnant à ce sujet un exposé assez détaillé des techniques de la chasse aux scorpions et des soins apportés à leurs piqûres. L'efficacité de la « médecine populaire », si elle est parfois reconnue par l'auteur et cautionnée par la médecine savante, n'en demeure pas moins étonnante à ses yeux comme le montre l'exemple de Ahmad al-kanafânî, un pâtissier guérisseur.

[Ahmad al-kanafânî] était un homme corpulent qui vendait des kunâfa à Bâb al-Mutawallî. Il portait un cafetan et un turban mais pas de jibba. II était célèbre pour la qualité de ses kunâfa et pour posséder un remède contre la lèpre et les maladies de peau. Un grand médecin rapportait que l'un de ses serviteurs était atteint de lèpre. Sa maladie s'aggrava, il lui prescrivit des remèdes qui demeurèrent inefficaces. Une vieille femme l'emmena chez [Ahmad al-kanafânî] et, étonnamment, il guérit complètement. Peut-être son remède était-il une sorte de pommade efficace pour cette maladie. Mais il est curieux que ce soit un pâtissier qui administre ce genre de traitement. (DC, «Al-sayyid Ahmad al-kanafânî », p. 241)

\section{Les remèdes relevant de la croyance}

D'autres remèdes sont présentés comme relevant de la croyance. Les femmes prêtent des propriétés abortives aux infusions des bourgeons de henné. Les Égyptiens croient que les "graines de baraka " (habbet al-baraka), soit les graines de la fleur de fenouil, soignent la plupart des maladies, et notamment les

22. Il s'agit ici plus vraisemblablement de la vitamine C comme cela a d'ailleurs été corrigé dans la dernière édition du Dictionnaire, cf. Amîn, 1999. 
maladies de poitrine ${ }^{23}$. Pour se rétablir, la parturiente consomme une boisson à base de racine de mughât (Glossostemon brugieri), qui est aussi offerte aux visiteurs. Des vertus fortifiantes sont également prêtées à l'eau du Nil. Ahmad Amîn signale que les Égyptiens croyaient que les pois chiches soignaient la migraine ainsi que de nombreuses maladies. Le barbier faisait une entaille dans le bras pour y placer un pois chiche. II plaçait par dessus un morceau de carton qu'il entourait d'un mouchoir : le pois chiche absorbait les résidus (fadalât) du corps. L'auteur ajoute avoir parfois vu son père recourir à cette pratique. II indique également que les Égyptiens prétendent (yaz'umûna) que la cervelle d'âne (mukhkh al-himâr) soigne les maladies rhumatismales. Ils estiment que la plante appelée barnûf, vendue le mercredi de Job, qui précède la fête de chamm al-nasîm, est celle que Job utilisa pour se soigner ${ }^{24}$. Ils la font macérer pour se laver avec ce jour-là et guérir de leurs maux. Ils estiment également protéger leurs enfants des maladies grâce à la circoncision. L'auteur note aussi que les paysans croient que le mech, un fromage fermenté, éloigne la gale.

L'auteur dénonce par ailleurs les «charlatans » prétendument magiciens qui récitent des incantations pour soigner des maux imaginaires. L'article sur les vers de nez en fournit un exemple.

Le commun du peuple (al-'awamm) prétend qu'il y a dans le nez de petits vers et que certaines personnes disposent d'incantations ('azâ'im) qui, s'ils les récitent en frottant le nez, en font descendre les vers. J'ai moi-même assisté à cela et j'en ai fait personnellement l'expérience. La plupart du temps, I'homme est un charlatan (dajjâl) qui a placé des vers dans sa manche. Par des mouvements dérobés, il fait descendre les vers de sa manche dans le nez de I'enfant et l'on croit ainsi qu'ils en sortent directement. Dieu est plus savant. (DC, « Dûdat al-anf », p. 200)

\section{Des traitements " invraisemblables " ou " délirants »}

D'autres traitements sont qualifiés $d^{\prime}$ « invraisemblables» ou « délirants » (khurâfí) et abordés sous l'angle de la superstition. Dans l'article "Al-gaza'» (terme signifiant à la fois une profonde tristesse et la nausée), I'auteur explique que les Égyptiens utilisent parfois pour soigner la nausée un « remède invraisemblable » qui consiste à accrocher un fétu de paille à sa coiffure pour le fixer du regard et concentrer ainsi son attention pour ne plus penser à son écœurement. ${ }^{25}$ Ahmad Amîn justifie la présence d'un article sur la migraine, « affection bien connue », par l'exposé des « traitements invraisemblables »

23. Les " graines de baraka », appelées aussi graines noires, sont également utilisées pour neutraliser l'effet du mauvais œil, Bachatly, 1929, p. 49-60.

24. Job fut frappé $d^{\prime}$ 'un « ulcère malin » des pieds à la tête et grattait ses plaies avec un tesson (Job, 1,7;2,8).

25. On trouve la description d'un procédé similaire, utilisant du papier jaune, dans l'article «Al-taqrîfa », p. 124. 
des « charlatans », soit la section des artères temporales pour que le mal s'en aille avec le sang ${ }^{26}$, le dessin avec de la salive d'une croix sur les tempes du malade et la récitation d'incantations magiques. Il signale également que certains Égyptiens se protègent du choléra par des moyens « invraisemblables » et relate qu'il a vu en pleine rue un homme juché sur une échelle et qui donnait des coups de ciseaux en l'air, prétendant ainsi tuer les microbes.

\section{Des remèdes inefficaces et meurtriers}

L'article "Al-hummâ » expose divers remèdes employés par le commun du peuple ('âmma) pour soigner la fièvre. L'auteur y oppose le savoir médical aux croyances du commun du peuple. Le premier traitement, la diète sans viande, est décrit d'après le propre témoignage de l'auteur : "Je fus une fois pris de fièvre et ils m'interdirent de manger de la viande, ne fût-ce qu'une fine tranche. Ils changèrent leur manière de cuisiner afin que l'odeur ne me tourmentât pas. De la sorte, je m'affaiblis et me sentais très fatigué. Le quatrième et le cinquième jours, je décidais de manger. Ils me présentèrent deux poulets bien gras et me préparèrent de la mulûkhiyya. Ils redoutaient de [me voir] manger mais, curieusement, je fus complètement guéri après ce repas » (DC, «Alhummâ », p. 181-182). Ahmad Amîn recouvrit donc la santé lorsqu'il cessa de suivre le traitement qu'on lui avait prescrit. Outre les amulettes, I'auteur mentionne que le commun du peuple soigne parfois la fièvre avec une pommade d'amidon et de vinaigre, ou une mouche de cheval, collée sur un morceau de pâte et que l'on force le malade à avaler. Il y indique aussi que l'on accroche parfois un os d'infidèle au cou du malade ${ }^{27}$. Il dénonce enfin le nombre de victimes causées par ce genre de remèdes. Dans cet article, I'auteur montre clairement que les remèdes apportés à la fièvre sont inefficaces et meurtriers. Avec I'utilisation des mouches et d'ossements humains, il illustre également combien ils sont répugnants et dégradants.

En dépit du cadre historique que l'auteur donne à son étude, puisqu'il s'agit de consigner des traditions sur le point de disparaître à l'attention des enfants des nouvelles générations, la place importante accordée aux thèmes de la santé et de l'hygiène témoigne de préoccupations plus actuelles face à la situation sanitaire de l'Égypte. Des généralisations optimistes sur l'adduction d'eau, I'utilisation d'antiseptiques et des médicaments modernes ont sans doute conduit Ahmad Amîn à reléguer dans le passé certains maux et divers comportements.

26. Le recours à la saignée contre les maux de tête, opérée par le barbier qui pratique une série d'incisions sur les tempes ou le front du malade, est signalé par de nombreux auteurs.

27. Un usage similaire est décrit par Edward Lane, qui note que, parmi les remèdes " imaginaires » utilisés contre la fièvre, certaines femmes portent autour du cou le doigt desséché d'un juif ou d'un chrétien, Lane, 1989, p. 261. 
II met principalement en cause la représentation du corps, dont les organes et le fonctionnement lui paraissent méconnus. Support de présages et soumis à l'emprise des esprits, le corps humain semble largement dominé par des influences surnaturelles. Ahmad Amîn déplore également I'ignorance de I'étiologie des maladies. Avec les djinns et le mauvais œil, I'attribution d'une origine surnaturelle implique le recours à des traitements magiques comme le zâr, les incantations et les amulettes. L'auteur oppose des explications scientifiques à de telles représentations. Il met notamment en évidence le mécanisme de la contagion des maladies dues aux microbes, à la contamination de l'eau, au manque de propreté et à l'ignorance des règles élémentaires d'hygiène. Et ce sont notamment les principes de l'hygiène qui paraissent introduire une véritable rupture avec la médecine ancienne, selon laquelle la santé dépend avant tout des aliments, du climat et du tempérament (voir Chiffoleau, 1994, p. 421). En opposant le savoir médical aux croyances des Égyptiens, en énonçant des règles $d^{\prime}$ hygiène, cet ouvrage prend un caractère pédagogique qui témoigne de sa vocation à être un outil d'éducation populaire, comme le montre aussi le choix de la forme du dictionnaire, un genre instructif et utilitaire. Ceci renvoie également à la mission d'éducateurs que se donnèrent les intellectuels vis-àvis de "leur " peuple, qu'ils devaient affranchir des "superstitions».

Dans bien des ouvrages traitant de la «médecine populaire », le simple catalogue des prescriptions médicales, présentées sans aucune explication, est censé démontrer leur caractère grotesque et absurde. Si étranges et imprégnées de magie qu'elles puissent paraître, les prescriptions médicales « traditionnelles » n'en reposent pas moins sur des savoirs naturalistes, souvent qualifiés d'empiriques, dont l'efficacité curative se voit parfois scientifiquement reconnue. Dans le Dictionnaire de Ahmad Amîn, le savoir que constitue la médecine " populaire » apparaît largement disqualifié. Rares sont les traitements dont l'efficacité est validée par l'auteur, avec la caution des médecins ou de la science moderne. Nombre de remèdes sont décrits comme des croyances voire des superstitions. Si la présentation des maladies et des pratiques thérapeutiques donnée par Ahmad Amîn peut former un « cas d'école » du discours réformiste en la matière, il est intéressant de le trouver à l'œuvre dans un dictionnaire des traditions et des coutumes, où il participe à la définition même de l'objet du folklore. Celle-ci suppose ici la disqualification et la marginalisation de la culture dite populaire (Certeau, 1993). On peut ainsi voir dans le discours savant qui s'élabore sur la «culture populaire » I'expression d'une position dominante et l'exercice $d^{\prime}$ 'une certaine forme de normalisation culturelle. L'épuration de la culture populaire permet également son appropriation. Ainsi que remarque Philippe Martel au sujet du rapport de l'abbé Grégoire sur la nécessité et les moyens d'anéantir les patois, il s'agit bien " d'autopsier » un objet : «On tue les patois, mais c'est pour mieux détrousser leurs cadavres » (Martel, 1997, p. 3 520).

Les pratiques thérapeutiques traditionnelles ou populaires sont ainsi consignées pour être dénoncées. Si elles éveillent une certaine curiosité 
ethnographique, elles sont abordées dans une intention réformatrice : il faut " observer pour former » (Ozouf, 1981, p. 229), " collectionner » et « réduire » (Certeau, 1993, p. 50). L'approche critique et éducative qu'adopte l'auteur montre le caractère ambivalent de l'objet du folklore, qui se construit à la croisée des descriptions ethnographiques et des projets de réforme sociale, telle une science appliquée, le savoir sur le peuple devant également fournir les moyens de son éducation.

\section{INDEX DES ARTICLES}

«Al-ibra » (I'aiguille), 1, p. 1-2 (90 lignes)

"Ibn al-balad», 5, p. 6-8 (91 lignes)

«Al-Atrâk» (les Turcs), 7, p. 23-24 (94,5 lignes)

«Al-ahjiba » (les amulettes), 9, p. 25 (36 lignes)

«Al-ad'iya » (les prières, les invocations), 11, p. 28 (21 lignes)

«Arbi'â' Ayyûb» (le mercredi de Job), 13, p. 29 (6,5 lignes)

«Al-Azhar » (al-Azhar), 17, p. 30-33 (144,5 lignes)

"Istihdâr al-arwâh» (I'invocation des esprits), 19, p. 35-36 (45 lignes)

«Al-usra » (la famille), 24, p. 38-40 (125 lignes)

« Ism al-tafdîl » (le comparatif), 26, p. 42-44 (95,5 lignes)

«Al-quchlâq »(I’hôpital), 30, p. 47 (8,5 lignes)

«Ashâb al-'âhât » (les infirmes), 32, p. 48 (17,5 lignes)

"Istabl 'Antar» (d'anciens tombeaux de l'époque pharaonique ; la chasse aux scorpions), 33, pp. 48-49 (34 lignes)

«Al-a'râb »(les bédouins), 34, p. 49-50 (59 lignes)

«Al-afyûm » (I'opium), 36, p. 51 (10 lignes)

"Aqdâm wa a'tâb wa nawâs » (les présages tirés des bêtes de somme, du seuil des maisons et des chevaux), 38, p. 52 (22 lignes)

«Al-amthâl »(les proverbes), 45, p. 61-70 (379 lignes)

«Al-amrâd » (les maladies), 46, p. 70-71 (34 lignes)

«Al-awqâf » (les biens de mainmorte), 53, p. 76 (36 lignes)

«Al-bâdhinjân » (l'aubergine), 55, p. 79 (23 lignes)

«Al-bakhûr »(I'encens), 58, p. 80-81 (38,5 lignes)

«Al-barâbra » (les Nubiens), 61, p. 82-83 (32,5 lignes)

«Al-barâghîth » (les puces), 63, p. 83-84 (18 lignes)

«Bard al-'agûz » (les jours les plus froids de l'hiver, les huit premiers jours du mois copte d'Amchîr), 65, p. 85 (6 lignes)

«Bartama » (baragouiner), 66, p. 85 (15 lignes)

«Al-baraka » (la baraka, la bénédiction), 68, p. 86 (25 lignes)

«Basal »(oignon), 73, p. 88-89 (25,5 lignes)

«Bat» (canard), 75, p. 89 (8 lignes)

« Batn» (ventre), 77, p. 90 (26,5 lignes)

«Battîkh » (pastèque), 78, p. 90-91 (28 lignes) 
«Al-baqq » (les punaises), 82, p. 92 (16,5 lignes)

«Al-balâ » (la syphilis), 86, p. 94 (11 lignes)

«Bunduqî »(sequin vénitien), 97, p. 97 (21,5 lignes)

«Bûza » (bière d'orge), 106, p. 104 (23 lignes)

«Al-bi'r» (le puits), 110, p. 105-106 (9,5 lignes)

"Al-tahiyyât » (les compliments, les formules de politesse), 121, p. 113-114

(18,5 lignes)

«Tarbiyat al-atfâl » (l'éducation des enfants), 124, p. 114-115 (30 lignes)

«Al-Tarbî'a » (un quartier du Caire), 125, p. 115 (16 lignes)

«Turmus » (lupin), 127, p. 116 (15,5 lignes)

"Taskhîr al-jânn » (asservir les djinns), 129, p. 116-119 (96,5 lignes)

"Al-tafâ'ul wa-l-tachâ'um » (les bons et les mauvais présages), 139, p. 124

(28 lignes)

«Al-taqrîfa » (la nausée), 140, p. 124 (5 lignes)

"Tilâwat al-qurân » (la récitation du Coran), 141, p. 124-125 (49,5 lignes)

«Al-jarab» (la gale), 152, p. 135 (18,5 lignes)

"Al-gaza' » (terme signifiant à la fois une profonde tristesse et la nausée), 156,

p. 137 (18 lignes)

« Jinn »(djinn), 165, p. 141-143 (117,5 lignes)

« Hagar al-kabbâs »(des pierres aux vertus médicinales et magiques), 179,

p. 155 (29,5 lignes)

« Hatt» (mettre, poser), 191, p. 171 (20,5 lignes)

« Halaq bilâ awdân » (expression, des boucles sans oreilles), 199, p. 175 (11 lignes)

« Al-hamâ » (la belle-mère), 201, p. 177 (22,5 lignes)

" Hamâm » (pigeon), 203, p. 178-179 (42 lignes)

«Al-hammâm »(le hammam), 204, p. 179-180 (48,5 lignes)

«Al-hummusa wa-l-kayy bi-I-nâr » (cautérisation par le feu et application d'un pois chiche dans une entaille faite sur la peau), 205, p. 180 (18 lignes)

«Al-hummâ » (la fièvre), 208, p. 181-182 (32 lignes)

"Hinna » (henné), 210, p. 182-183 (31,5 lignes)

"Al-khitân » (la circoncision et l'excision), 215, p. 187-189 (60 lignes)

«Al-khass» (la laitue), 221, p. 192 (25 lignes)

"Al-khalîg » (I'ancien canal du Caire), 229, p. 195 (17,5 lignes)

«Al-khamâsîn » (le vent de sable), 230, p. 195 (10 lignes)

«Al-khawâga » (I'étranger, I'Européen), 232, p. 196 (35,5 lignes)

" Dustûr »(diverses expressions), 237, p. 199-200 (14,5 lignes)

«Dûdat al-anf »(le ver de nez), 240, p. 200 (8 lignes)

«Al-ruqa » (la médecine populaire, les remèdes de bonne femme), 253, p. 214

(7,5 lignes)

"Sabâris » (les ramasseurs de mégots), 267, p. 227 (15,5 lignes)

"Al-subû' " (fête qui a lieu au septième jour de la naissance d'un enfant), 270,

p. 229 (26,5 lignes)

« $\mathrm{Al}$-saratân »(un ver parasite, le cancer), 275, p. 231 (9 lignes) 
«Al-sultân Salîm » (le sultan Sélim Ier), 282, p. 234-237 (155 lignes)

«Suwar al-qurân » (les propriétés des sourates du Coran), 286, p. 239-240

(34 lignes)

«Al-sûq » (le souk, le marché), 287, p. 240 (24,5 lignes)

"Al-sayyid Ahmad al-kanafânî » (Ahmad al-kanafânî, un pâtissier guérisseur), 289 , p. 241 (11 lignes)

«Al-Chahhâdhûna » (les mendiants), 295, p. 246-247 (74 lignes)

"Al-Charbât »(les sirops), 296, p. 247-248 (16 lignes)

«Al-Charkas» (les Circassiens), 298, p. 248 (28 lignes)

"Al-chuhûr al-qubtiyya » (le calendrier copte), 304, p. 253-254 (46,5 lignes)

"Al-sudâ'» (la migraine), 308, p. 260 (8 lignes)

«Al-sinâ'a al-misriyya » (I'industrie égyptienne), 311, p. 262-263 (43,5 lignes)

«Al-dabba » (la serrure en bois), 313, p. 267 (32 lignes)

«Al-darâ'ib » (les impôts), 314, p. 267-268 (52,5 lignes)

«Tâsit al-khadda » (le « bol d'effroi »), 319, p. 275 (12 lignes)

"'Abduh wa Almaz» (un couple de chanteurs célèbres), 326, p. 283-284

(60,5 lignes)

«Al-'ajâ'iz » (les vieilles femmes), 327, p. 284 (16 lignes)

" 'Iddiyyat Yâ Sîn » (la récitation de la sourate Yâ Sîn), 328, p. 284 (14,5

lignes)

«Al-'achba » (la salsepareille), 330, p. 285 (7 lignes)

"Al-'aqîq » (la cornaline), 333, p. 286 (5,5 lignes)

" 'Ayyin al-sîra » (une source près du tombeau de l'imam al-Chafî̀î), 348,

p. 294 (10 lignes)

«Al-fiqî » (celui qui récite et enseigne le Coran), 367, p. 308 (16 lignes)

«Al-fallâh » (le paysan), 369, p. 310-311 (52,5 lignes)

«Al-qarr » (le mauvais œil causé par les bavardages des gens), 379, p. 319 (7

lignes)

«Al-qubla » (le baiser), 378, p. 318-319 (40,5 lignes)

"Al-qurâ'» (la teigne), 383, p. 322 (25 lignes)

«Al-qur’a / al-tajnîd / al-jihâdiyya » (la conscription), 385, p. 323 (39 lignes)

«Qasab» (canne à sucre), 390, p. 325-326 (33 lignes)

"Al-qaml wa-l-burghûth wa-l-ba'ûd wa-l-baqq» (les poux, les puces, les moustiques et les punaises), 396, p. 328 (17,5 I.)

"Qiyâs al-athar » (un procédé divinatoire pour prédire la guérison d’un malade

à partir d'un de ses effets personnels), 398, p. 329 (9 lignes)

« Kubba » (abcès, bubon), 402, p. 334 (5,5 lignes)

«Al-kuttâb » (I'école coranique), 404, pp. 334-335 (57 lignes)

«Al-kuhl »(le khôl), 406, p. 336 (18 lignes)

"Al-kursî » (la chaise), 407, p. 336 (26,5 lignes)

«Al-kinâyât » (les expressions imagées), 412, pp. 339-340 (26 lignes)

«Al-kûlîrâ » (le choléra), 413, p. 340 (16 lignes)

«Al-lubân el-dhakar» (le ladanum, une résine tirée du ciste), 416, p. 345

(15,5 lignes) 
«Al-laymûn al-saghîr » (le petit citron), 423, p. 350 (18,5 lignes)

«Al-ma'kûlât al-khâssa » (les aliments particuliers), 425, p. 353-354 (34 lignes)

«Al-mâl al-haram » (I'argent mal acquis), 426, p. 354 (24 lignes)

« Al-muhtasib» (I'inspecteur des marchés), 431, pp. 356-357 (41,5 lignes)

"Mukhkh al-himâr » (la cervelle d'âne), 435, p. 361 (2,5 lignes)

"Al-mekhallilâtî » (le vendeur de légumes confits au vinaigre), 436, p. 361 (20 lignes)

"Al-misahharâtî » (celui qui, en passant dans les rues, réveille les gens avant

I'aube durant le mois de ramadan), 442, p. 366-367 (19,5 lignes)

«Al-mech » (un fromage fermenté), 443, p. 367 (21,5 lignes)

«Al-machrûbât » (les boissons), 444, p. 367-369 (76 lignes)

"Al-ma'gûn » (drogue composée d'un mélange de haschich, d'opium, de plantes et d'épices), 449, p. 371-373 (66, 5 lignes)

«Al-muqawwiyât » (les fortifiants), 457, p. 376 (9 lignes)

"Al-mamâlîk » (les mamelouks), 466, p. 380-381 (49 lignes)

« Mîzâniyyat al-bayt » (le budget de la maison), 476, p. 390-391 (42 lignes)

«Al-mayya » (I'eau), 477, p. 391 (19 lignes)

«Al-mîda » (le bassin d'ablution), 478, p. 391-392 (26 lignes)

«Al-nidâ » (les appels des vendeurs ambulants), 481, p. 395 (13,5 lignes)

«Al-nudhûr » (les ex-voto, les offrandes votives), 482, p. 396 (29,5 lignes)

«Al-nazâfa » (la propreté), 486, p. 397-398 (26,5 lignes)

"Al-nuqta » (une fête liée à la crue du Nil (lîlit al-nuqta), désigne également une somme d'argent offerte aux mariés, le pourboire donné aux almées et les cadeaux offerts lors d'une circoncision, d'un mariage ou du pèlerinage), 489, p. 399-400 (24 lignes)

«Al-Nûbiyyûna »(les Nubiens), 491, p. 400 (22 lignes)

" Namâdhij » (modèles), 492, p. 401-404 (161 lignes)

«Al-Nîl » (le Nil), 493, p. 404-406 (87,5 lignes)

"Al-wiqâya » (la protection contre le mauvais œil), 505, p. 415 (16,5 lignes)

«Yâ farag » (les chasseurs de serpents), 508, p. 419 (12 lignes)

\section{RÉFÉRENCES BIBLIOGRAPHIQUES}

Amîn Ahmad, s.d., Hayâtî, Le Caire, Maktabat al-nahda al-misriyya, $7^{e}$ édition (1 ${ }^{\text {re }}$ édition 1950), $357 \mathrm{p}$.

- 1953, Qâmûs al-'âdât wa-l-taqâlîd wa-l-ta'âbîr al-misriyya, Le Caire, Lajnat al-ta'lîf wa-l-tarjama wa-l-nachr, 480 p.

- 1999, Qâmûs al-'âdât wa-l-taqâlîd wa-l-ta'âbîr al-misriyya, édition présentée et annotée par Muhammad al-Jawharî, Le Caire, wizârat al-thaqâfa, al-majlis al-a'lâ li-l-thaqâfa, 553 p.

Ayrout Henry Habib S. J., 1938, Mours et coutumes des fellahs, Payot, $187 \mathrm{p}$. 
BesançOn J., 1977, «Portrait de l'Égypte rurale au milieu du XXe siècle » dans Aulas M. C., Besançon J. et al., L'Égypte d'aujourd'hui. Permanences et changements 1805-1976, Paris, Éditions du CNRS, p. 179-210.

BaChatly Charles, 1929, "Notes sur quelques amulettes égyptiennes », Bulletin de la Société royale de Géographie d'Égypte, tome XVII, $1^{\text {er }}$ fascicule, p. 49-60.

Blackman, Winifred, 1948, Les Fellahs de Haute-Égypte, Vie religieuse, sociale et économique, le présent et les survivances anciennes, traduction de Jacques Marty, Paris, Payot, 249 p.

Boustani Rafic et Fargues Philippe, 1990, Atlas du monde arabe, Paris, Bordas, $144 \mathrm{p}$.

Bovier-Lapierre R. P., 1948, "Rapport du R. P. Bovier-Lapierre sur le Musée ethnographique », Extrait des procès-verbaux du Conseil d'administration, séance du 26 mai 1941, Bulletin de la Société royale de Géographie, tome XXII, fasc. 3-4, p. 183.

Certeau, Michel de, 1993, en collaboration avec Julia Dominique et Revel Jacques, "La Beauté du mort », La culture au pluriel, Paris, Seuil, 3 e édition

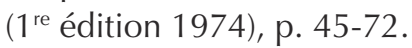

Chiffoleau Sylvia, 1994, "La réforme par I'hygiène. Une formule pour médicaliser les campagnes » dans Roussillon Alain (dir.), Entre Réforme sociale et mouvement national. Identité et modernisation en Égypte (1882-1962), Le Caire, Cedej, p. 421-441.

- 1997, Médecines et médecins en Égypte. Construction d'une identité professionnelle et projet médical, Paris, Éditions L’Harmattan, 334 p.

Collet Isabelle, 1987, «Les premiers musées d'ethnographie régionale en France » dans Musée national des arts et traditions populaires, Muséologie et ethnologie, Paris, Éditions de la Réunion des Musées nationaux, p. 68-99.

Henein Nessim Henry, 1988, Mâr Girgis, Le Caire, IFAO, 443 p.

Hınds Martin et Badawı El-Said, 1986, A Dictionary of Egyptian Arabic ArabicEnglish, Beyrouth, Librairie du Liban, 981 p.

Lane E., 1989, An Account of the Manners and Customs of the Modern Egyptians, Londres, East-West Publications [1 ${ }^{\text {re }}$ édition 1836], 583 p.

Lenclud Gérard, 1987, « La tradition n'est plus ce qu'elle était... Sur les notions de tradition et de société traditionnelle en ethnologie », Terrain, n 9, p. 110-123.

- 1990, «Vues de l'esprit, art de l'autre. L'ethnologie et les croyances en pays de savoir », Terrain, $n^{\circ} 14$, p. 5-19.

Martel Philippe, 1997, "Le Félibrige », Les lieux de mémoire, sous la direction de Nora, Pierre, tome 3, Paris, Gallimard, p. 3 515-3 552.

Ozouf Mona, 1981, "L'invention de l'ethnographie française : le questionnaire de l'Académie celtique », Annales E.S.C., 36 année, p. 210-227.

TADIE Arlette, 2002, Le Sel de la conversation. 3000 proverbes d'Égypte, Paris, Maisonneuve et Larose, 616 p. 